\title{
Meeting Report:
}

\section{AAPS-NIFDC Joint Workshop on Dissolution Testing, Biowaiver, and Bioequivalence}

e-mail:xujin.lu@bms.com

Xujin $\mathrm{Lu}^{1,{ }^{*},}$ Vivian A. Gray², Nikoletta Fotaki ${ }^{3}$, Jian-Hwa Han ${ }^{4}$, Johannes Krämer ${ }^{5}$, and Erika Stippler ${ }^{6}$

${ }^{1}$ Bristol-Myers Squibb Company, New Brunswick, NJ, USA

${ }^{2}$ V. A. Gray Consulting, Hockessin, DE, USA

${ }^{3}$ University of Bath, Bath, UK

${ }^{4}$ Abbvie, Inc., North Chicago, IL, USA

${ }^{5}$ PHAST GmbH, Homburg, Germany

${ }^{6}$ U.S. Pharmacopeia, Rockville, MD, USA

A joint workshop of the American Association of Pharmaceutical Scientists (AAPS) and the Chinese National Institutes for Food and Drug Control (NIFDC) on dissolution testing, biowaiver, and bioequivalence took place in Tianjin, China, from June 21 to $22,2016$.

The AAPS and NIFDC cosponsored this event. The organizing institutions were the AAPS In Vitro Release and Dissolution Testing (IVRDT) Focus Group, Chinese Journal of New Drugs, and Tianjin Institute for Drug Control, supported by the Medical Instrument Branch of China Instrument and Control Society and the China Pharmaceutical Enterprise Development Promotion Association. The goals and objectives of the workshop were: (1) enhance the communication among Sino and American industry scientists, regulators, and academics in the field of in vitro and in vivo drug release and the use of dissolution as a tool for drug development, biowaiver, and bioequivalence studies; (2) share knowledge in dissolution testing, apparatus calibration, bioequivalence application, and drug specification settings; (3) disseminate regulatory and compendial information to facilitate method development, current Good Manufacturing Practice compliance, and quality control (QC) of generic products; and (4) establish networking and collaboration among scientists from the drug control and inspection agencies, academia, and the pharmaceutical industry.

The workshop was co-chaired by Dr. Xujin Lu (IVRDT Focus Group) and Dr. Baoming Ning (NIFDC). Members of the Organizing Committee were Ms. Vivian Gray (V. A. Gray Consulting), Ms. Lan Lin (NIFDC), Professor Qiming Zhang (NIFDC), Professor Jianqiang Shao (Tianjin Institute for Drug Control), Mr. Dawei Liu (Chinese Journal of New Drugs), and Mr. Yucheng Gao (China Instrument and Control Society).

\section{DAY 1}

\section{Session 1}

In his opening remarks, Dr. Xujin Lu reiterated the goals of the workshop. Ms. Lan Lin (Director of Chemical Drugs Division, NIFDC) expressed strong support from the Chinese co-sponsor and organizing institutions and introduced the first session.

Professor Nan Nan (NIFDC) delivered the first presentation titled "Application of Dissolution Testing in Biorelevance Evaluation of Generic Drugs." Following recent publications by China Food and Drug Administration (CFDA) on \#106-2016, "Guidance for Bioequivalence Evaluation of Generic Drugs," and \#61-2016, "Guidance for Measurement and Comparison of Dissolution Profiles for Solid Oral Dosage Drugs," Professor Nan elaborated the requirements of these guidelines. She addressed the scope of Guidance \#61, the objective of the dissolution profile studies, the dissolution profile measurement, the dissolution profile comparison and similarity, and a case study.

Professor Nan indicated that Guideline \#61 is to formalize the current studies and work in China using in vitro dissolution for the bioequivalence evaluations of generic drugs in solid oral dosage forms, which is an integral part of executing Guidance \#106. An in vitro dissolution profile, as opposed to a single point of drug release, demonstrates the entire process of drug release that may provide relevant information on drug in vivo performance and, therefore, could be a simple and direct tool for formulation development, batch-to-batch consistency evaluation, and QC for formulation and process change. Studies of dissolution profiles will be beneficial to learn the characteristics of a comparator or branded drug, to apply the learning to evaluating the quality of a generic 
drug versus branded drug, to guide a bioequivalence study, and to establish a reliable dissolution testing method. Professor Nan provided detailed instructions on the measurement of dissolution profiles and development and validation of dissolution methods. She recommended first finding the compendial methods that have been published in USP, FDA dissolution database, Japanese Orange Book, and the other pharmacopeia. She also indicated it is not realistic to establish IVIVC dissolution methods for every drug product. However, it is possible to develop a dissolution method that can be used for $Q C$ and drug inspection through differentiation of the changes in formulation, process, and batch-tobatch consistency. For dissolution profile comparisons, Professor Nan recommended using Similarity Factor $f_{2}$ over other non-model dependent methodologies. In addition, she emphasized the conditions that have to be met when using the $f_{2}$ method. Professor Nan described a case study in great detail. Terazosin Hydrochloride (TH) is a highly soluble drug in BCS Class I or III. Compendial methods for the tablet dissolution can be found in FDA database, Japanese Orange Book, USP 38, as well as CHP 2015. A method was determined for the comparison of a branded TH tablet product made by Abbott with a generic TH tablet product, which utilized four different dissolution media: water, $0.1 \mathrm{~N}$ hydrochloric acid, $\mathrm{pH}$ 4.0 and pH 6.0 buffers, and six sampling time points. A comparison between the average dissolution profiles from 12 dosages of the reference TH product and the generic $\mathrm{TH}$ product passed the $f_{2}$ test and therefore demonstrated in vitro similarity.

Dr. Utpal Munshi (Division of Bioequivalence I, Office of Generic Drugs, CDER, FDA, USA) gave the second talk, entitled "Dissolution: A Critical Tool in the Evaluation of Generic Drug Product." Dr. Munshi started with a question, what do we use dissolution for? From the perspective of drug product evaluation, he said the answer is for the QC of the finished dosage form, a tool to assess waiver requests of the in vivo bioequivalence study requirement, to support post-approval changes, to assess dumping of dose from the product, and as part of a weight-of-evidence approach to assessing bioequivalence (BE). He elaborated each of the applications throughout his talk.

As a method to control the quality of the finished dosage form, the products where the $Q C$ dissolution method is employed include solid oral dosage forms, oral suspensions, transdermal products, and injectable suspensions. The assessment of biowaiver requests was addressed from several aspects. For immediate-release
(IR) products, he pointed to 21 CFR 320.22(b)(2) that states one of the requirements for biowaiver is "both drug products meet an appropriate in vitro test approved by FDA." Telmisartan tablets provided an example. FDA compares the dissolution profile data of the waiver strengths (typically the lower strengths) with the strength of the batch used in the pivotal BE study. If dissolution profiles are "similar," dissolution is considered to be supportive of the waiver request. Assessing biowaiver for modified-release (MR) products is not a "waiver." Rather, as stated in 21 CFR 320-24(b)(6), "Any other approach deemed adequate by FDA to measure bioavailability or establish bioequivalence," which usually requires proportional similarity, an understanding of the drug release mechanism, and a similar dissolution approach to that used in IR products (with the exception of multimedia testing with different $\mathrm{pH}$ values). For Drug Efficacy Study Implementation (DESI) products, Dr. Munshi described the biowaiver with the example of the Acetaminophen/Butalbital/Caffeine capsule, which is a DESI-effective drug and exempted from in vivo BE study. For a Biopharmaceutics Classification System (BCS)-based biowaiver, he explained why waivers can be based on solubility, permeability, and dissolution, and clarified that only IR solid oral dosage forms with a BCS Class I drug substance that exhibit rapid dissolution or a BCS Class III drug substance that exhibit very rapid dissolution are eligible for waiver. The others, like MR drug products, narrow therapeutic range drugs, and dosage forms intended for absorption in the oral cavity are exempt from waiver.

Dissolution can be used to support post-approval changes. Dr. Munshi explained the general concepts of related FDA guidances for Scale-Up and Post Approval Changes of IR and MR. Changes in the formulation and manufacturing are classified into different levels depending on the extent of the change from the originally approved product. The nature and number of studies needed depend on the level of the change. Comparative dissolution is often used to support a post-approval change. In some cases, an in vivo $B E$ study may be recommended to support the postapproval change. In lieu of an in vivo study this case, an in vitro-in vivo correlation (IVIVC) may be used to support the change.

To assess the dumping of dose from the product, dissolution has been playing an important role. He described the requirement for in vitro alcohol dosedumping testing, and indicated if the test $(T)$ and reference (R) products do not behave in the same way in the presence of alcohol, the safety/efficacy profile 
may differ between $T$ and $R$, causing concerns about the therapeutic equivalence. He also listed four drug products where alcohol dose dumping is currently requested.

Another application of dissolution is as part of a weightof-evidence approach to assessing BE. He listed some example cases and presented details for three cases. First, he discussed ergocalciferol capsules that contain the active pharmaceutical ingredient (API) in "edible vegetable oil." An in vitro approach may suffice provided control of the formulation similarity between the test and reference products. The in vitro approach is a specialized type of dissolution test known as Quantitative Capsule Rupture Testing, which can assess BE provided that the $T$ and $R$ products are qualitatively and quantitatively the same. Secondly, he discussed vancomycin $\mathrm{HCl}$ capsules, a locally acting drug product that is poorly absorbed after oral administration but is solubilized and transported by gastrointestinal (GI) fluid to the site of action in the lower GI tract. The BE can be assured when the effect of the excipients on transporting the drug and on the effectiveness of the drug at the site of action are similar between the $T$ and $R$ products. Also, the release of vancomycin from the $T$ and $R$ is equivalent, e.g., similar $T$ and $\mathrm{R}$ dissolution at all physiologically relevant $\mathrm{pH}$ ranges. Third, he discussed cyclosporine ophthalmic emulsion to reduce regulatory burden and regulatory risk. Two options can be adopted to demonstrate BE for this product. One is an in vivo option using a clinical endpoint study. The other option is an in vitro option consisting of two parts: (1) a comparative study on globule size distribution and other comparative physicochemical studies and (2) a comparative in vitro drug release rate test that can discriminate the effect of process variability in the production of the test formulation. In conclusion, Dr. Munshi emphasized the importance of dissolution to all the applications and provided references to FDA guidances and published documentation.

Dr. Erika Stippler (U.S. Pharmacopeia, USA) gave a presentation titled "USP Compendial Dissolution/Drug Release Testing-Overview and Update." Dr. Stippler compiled the USP General Chapters relevant to the performance testing of various dosage forms and presented the recent changes and updates on each of these chapters.

The USP General Chapters <701> Disintegration, <711> Dissolution, and $<724>$ Drug Release are mandatory chapters and describe test apparatus for the evaluation of the performance of various dosage forms. Whereas dissolution Apparatus 1 and 2 are well-established test apparatus and are widely used in each phase of a Dissolution

Technologies NOVEMBER 2016 formulation's life cycle, the other dissolution apparatus still find limited use. These limitations can be due to the apparatus design or due to limited experience in applicability and use of those apparatus.

Dr. Stippler presented the most recent changes in General Chapter $<711>$ being effective in USP 39-NF 34. This refers to the dissolution tests of hard and soft gelatin capsules and gelatin-coated tablets, which do not conform to the dissolution specifications in the specific drug product monograph. In those cases, the dissolution test can be now performed with the addition of different enzymes depending on the $\mathrm{pH}$ value of the dissolution medium.

Several information chapters describing the performance tests of various dosage forms other than those for solid oral dosage forms have been developed in recent years. General Chapter <1724> Semisolid Drug ProductsPerformance Tests became official in USP 37-NF 32 (May 01, 2014) and describes three apparatus for drug release testing of semisolid dosage forms. The official revision of the General Chapter <1094> CapsulesDissolution Testing and Related Quality Attributes (USP 37-NF 31, Supplement 1, Aug. 01, 2014) provides detailed information on the specific challenges of crossed-linked gelatin capsules in the dissolution test, and describes approaches to resolve them. The revised General Chapter $<1092>$ The Dissolution Procedure: Development and Validation has become effective in USP 38-NF 33 Supplement 1 (Aug 01, 2015) contains very useful step-bystep recommendations on how to develop and validate dissolution procedures. The new General Chapter <1771> Ophthalmic Products-Performance Tests became official in USP 39-NF 34. The General Chapter <1090> Assessment of Drug Product Performance-Bioavailability, Bioequivalence, and Dissolution recently underwent a major revision, and the proposed new version of this chapter was published in Pharmacopeial Forum (PF) 42(4). Also, a new informational General Chapter <1004> Mucosal Drug Products-Performance Tests has been published in PF 41(5).

Dr. Stippler concluded her presentation presenting the USP's Dissolution database accessible online. This database is a searchable list of all dissolution/drug release test procedures described in the USP drug product monographs.

The next speaker was Ms. Vivian Gray, whose talk was titled "Differences and Harmonization of CMC Area of Dissolution, EU/US Perspectives." This talk began with a description of the successful harmonization of the FDA BCS Biowaiver guidance with the other global guidances. 
The talk focused on the reduction of the $90 \%$ permeability criteria to $85 \%$, the allowance for 75 -rpm paddle speed; the inclusion of BCS Class III to be eligible for a biowaiver given very rapid dissolution ( $85 \%$ dissolved in $15 \mathrm{~min}$ ) in all three prescribed media; the $\mathrm{pH}$ range reduced to 1.2 to $6.8 \mathrm{pH}$; and the volume of media was reduced to $500 \mathrm{~mL}$. She highlighted the differences and similarities in the USP and the European Pharmacopeia regarding dissolution topics. There was a discussion of the elements that are found in the USP $<711>$ Dissolution General Chapter that are not present in the European Pharmacopeia. These elements are the Performance Verification Test (PVT), enzyme addition for cross-linking, pooled sampling, and 2- to 4-L vessels. Ms. Gray gave an account specifically regarding $f_{2}$ criteria and rules that are different or slightly different in difference regions and in the many guidance documents.

\section{Session 2}

The second session began in the afternoon with Ms. Vivian Gray giving an overview of the AAPS, the U.S. organization that co-sponsored the event. She highlighted the availability of e-membership for Chinese scientists. She told the audience about the new eLearning course on dissolution.

The next speaker, Dr. Johannes Krämer (PHAST $\mathrm{GmbH}$, Germany), presented a talk titled "Predictive Dissolution Testing for Development of New Drugs." Dr. Krämer started with a clarification-the results of in vitro dissolution testing may be used as a surrogate of bioequivalence testing to prove a similarity of dosage forms. However, dissolution testing alone does not allow for the prediction of oral bioavailability of an oral drug product. Dissolution testing does not provide an absolute measure of bioavailability, rather, it allows for relative estimates of bioavailability changes and can reveal relative differences in formulation variations.

The goal of predictive dissolution testing is to evaluate the in vitro performance of dosage forms. Dr. Krämer indicated that, in order to assign any results clearly to the impact of the dosage forms, preliminary testing should cover the physicochemical properties of the drug substance, given that drug substance characteristics may be leveled out or amplified or masked by technological means.

In the lifecycle of drug products, in vitro dissolution testing may have different goals. The general requirement is to predict changes of bioavailability as the surrogate of the therapeutic efficacy. In early development, the discriminatory power of in vitro methods is required. At that stage, different dosage forms may be evaluated. In vitro methods are expected to be sensitive to the dosage form properties. In the subsequent development phase, discriminatory power is, again, required, whereas the focus of in vitro testing is on the formulation differences (e.g., differences caused by changes in the composition). Also, sensitivity to variations in the manufacturing process with critical influence on the dosage form's in vivo performance is demanded. Once the drug product is registered and the market supply has started, the discriminatory power of dissolution methods is, again, required. At that stage, dissolution testing as a part of $Q C$ provides the proof of similarity of released lots to those lots included in early bioavailability (BA) studies and for which safety and efficacy data are available. Intra-lot homogeneity and lot-to-lot conformity are the quality parameters. Specifications of dissolution testing may be based on in vivo bioequivalence findings, providing that all batches "in spec" are bioequivalent. With that in mind, there is an ongoing scientific debate on whether predictive in vitro performance testing should reflect the in vivo dissolution, which takes place in the human $\mathrm{Gl}$ tract, or whether it should reflect the composite kinetics as it is observed from blood concentration analyses. In both cases, the likelihood of predicting the in vivo performance is assigned to in vitro experimental designs that are based on the most relevant in vivo conditions, such as $\mathrm{pH}$ value, surface-active ingredients, and lipids. This is of great importance in the case of evaluating IR dosage forms. For those extended-release (ER) dosage forms, which are designed to release the drug independently from the physicochemical surroundings of the human GI tract, in vitro simulation of in vivo conditions may be less important.

According to Dr. Krämer, predictive dissolution may be linked to biorelevant methods. The term biorelevant may have different meanings, depending on whether the API is for local or systemic application. The use of biorelevant media, as first described by Dressman et al., may be beneficial. Besides the composition of dissolution media, which has a great influence on the solubility of the drugs substance and, hence, on its dissolution rate, their volume is an important experimental detail. In the fasted state, physiologically available volumes are smaller than the typically used $900 \mathrm{~mL}$ of the classical USP paddle, Apparatus 2. However, an excessive volume may create conditions that mimic the in vivo absorption. To complete the dissolution medium list of functions, the transfer of mechanical energy from the stirring element to the surface of the specimen, at the given viscosity, is worth noting. Provided that in vivo dissolution is the 
rate-limiting step in the composite pharmacokinetic (PK) process, a relationship between parameters of in vitro dissolution kinetics and the rate and amount by which the active moiety is absorbed from the dosage form (i.e., its bioavailability) may be established.

The next speaker was Dr. Jian-Hwa Han (AbbVie Inc.) whose presentation was titled "Dissolution Method Development for New Drugs in Solid Dosage Forms According to FDA Biopharmaceutics' ExpectationsCase Studies." Dr. Han discussed the new considerations for dissolution method performance according to FDA's expectations. After years of discussions and debates on the dissolution method deliverables, the industry is still not clear on the direction for developing dissolution methods appropriate for their intended use. FDA's position is, very clearly, to guard the quality of every product for its safety and efficacy. FDA expect dissolution methods to be discriminatory and clinically relevant. The discriminating power may be verified by varying the critical materials attributes (CMAs) and the critical process parameters (CPPs). To confirm the method and specifications as clinically relevant, it requires collaborations among multifunctional areas (i.e., analytical, formulation, and PK groups). The formulators may have to make samples outside the normal design space and perform biostudies to evaluate the impact on in vivo performance. This information is critical for dissolution method development and for setting the appropriate specifications.

Three strategies were presented in this talk. The method development strategies were based on the BCS classifications and formulation design. Strategy A is for BCS Class I and III drugs with rapid drug release formulation that can follow FDA Draft Guidance (2015) and use the standard method with standard specification. Strategy B is designed for non-rapid drug releasing IR formulations, which covers all BCS class drugs. It is recommended to explore the dissolution performance in media with various pHs, using different apparatus, and different agitation rates to discover the proper discriminating power of the method. Strategy C is for ER formulation, which includes the preparation for an IVIVC in additional to all the practices applied to Strategy B.

The presentation also covered how to use formulation/ process design of experiment (DOE) to verify the method's discriminating power for setting up proper specification based on the clinical outcome (if available). Several cases presented. One case covered not discriminating for dissolution method and no difference in vivo performance. Another case discussed overly discriminating dissolution method with no difference in vivo performance. A third case evaluated ranking order observed by dissolution and samples tested for $\mathrm{BE}$ and non-BE, and the last case discussed IVIVC.

The presentation concluded that a dissolution test to support clinically relevant acceptance criteria could not be developed by analytical or dissolution groups alone. A strong collaboration is required among analytical, formulation, and PK groups. The formulation group may have to specially prepare "unordinary samples" to examine the impact of various CMAs/CPPs on the drug release both in vivo and in vitro. The PK group needs to design biostudies and collect clinical data for those "unordinary samples" as necessary. The absorption profile should be established for the studied product to serve as the reference profile for dissolution method development. The analytical group should select the most appropriate dissolution method conditions to match the "target/reference dissolution profile." They should also compare the dissolution profiles of those "unordinary samples," and set relevant acceptance criteria. The team will consolidate all the findings/learnings and define the "control space" for the manufacturing process (control strategy), which could assure the quality of the product.

Dr. Chunmeng Sun (China Pharmaceutical University) presented on behalf of Professor Jiasheng Tu (China Pharmaceutical University) who could not attend the meeting in person. The title of the presentation was "The In Vitro-In Vivo Correlation of Diclofenac Sodium Extended-Release Tablet." Dr. Sun presented a comprehensive study that used $\mathrm{pH}$-sensitive drug diclofenac sodium as a model drug to study the IVIVC of multi-sourced diclofenac sodium ER tablets. The in vivo part of the study was conducted using beagle dogs, with a random grouping, self-control, multi-agent, and multi-period crossover design. Diclofenac sodium tablet samples from five different generic sources were dosed and compared with a brand sample as a reference. Analytical methods were developed and validated for the analysis of the drug concentration in plasma. Results demonstrated that a significant difference in the PK profiles exist between the reference formulation and the test formulations, which indicated a risk that these generic formulations may not be bioequivalent to the reference. The relative bioavailability of each generic formulation also showed a significant difference from the reference product as demonstrated with a greater F-value. The in vitro dissolution tests were conducted by using several conditions. The devices used included a basket, paddle, and flow-through cell. The dissolution media were $\mathrm{pH}$ 4.0 and $\mathrm{pH} 6.8$ buffers, water, and fasted and fed state 
simulated intestinal fluid. Different agitation speeds, flow rates, and medium changes were also applied. Dissolution results were evaluated using both similarity factors $\left(f_{1}\right.$ and $f_{2}$ ) and Weibull parameter analysis (DDSolver). Two dissolution methods were identified that were capable of differentiating these formulations. Results from one method were demonstrated for the correlation of the in vitro release of diclofenac sodium with in vivo absorption in the beagle dogs. An evaluation was further conducted on the IVIVC under single dissolution conditions versus multi-dissolution conditions. A three-dimensional mathematic model was established that incorporated the $\mathrm{pH}$, time, and amount of drug release factors, which demonstrated better IVIVC and predictability.

Dr. Nikoletta Fotaki (University of Bath, UK) was the last speaker of the day with a presentation titled "Various Dissolution Apparatus in USP, such as Apparatus 4, with Application Case Studies." She started her presentation with a description of the four official dissolution apparatus for solid oral dosage forms (USP Apparatus 1, 2, 3, and 4) and highlighted their applications and points to consider during dissolution testing. The key features of the flow-through cell (Apparatus 4) were presented in detail. Case studies on the use of Apparatus 4 as a tool for setting conditions to ensure consistent in vitroin vivo performance of oral drug products and for the development of IVIVCs of a poorly soluble compound were presented. She concluded her presentation with a discussion on the points to consider in selecting dissolution apparatus based on the purpose of the dissolution test (QC vs research and development) and the drug and formulation properties.

\section{DAY 2}

\section{Session 3}

On the second day, the workshop started with Dr. Johannes Krämer with his talk titled "IVIVC According to USP General Chapter <1088> In Vitro and In Vivo Evaluation of Dosage Forms." Dr. Krämer began his talk describing the purpose of IVIVC. He stated that in vivo and in vitro evaluation of dosage forms may lead to a comparison of results first. It is desirable to establish a relation. In an ideal case, the results of a simple so-called QC dissolution test should ascertain the bioequivalence of all batches in specification. This would require the in vivo evaluation of dosage forms correlate to the in vitro evaluation of dosage forms by an IVIVC. With that goal in mind, the strategy in method development should include the evaluation of both the drug substance and the drug product. However, IVIVC is strictly dosage-form limited. This becomes obvious in the case of ER dosage forms, where the rate and extent to which the drug substance is absorbed may vary largely from dosage form to dosage form. Therefore, an IVIVC is primarily applicable for one product only.

To reach the goal of a predictive IVIVC in pharmaceutical development practice, the designs and methods of an in vivo bioavailability test provide fewer opportunities for modification and adjustment than the in vitro experimental design. Dr. Krämer explained this is due to the biological model, the related ethical constraints, and the regulatory requirements in addition to the extra time and effort to undertake an in vivo study. In vitro testing provides more room for variations. The common opinion is that the closer the in vitro setting mimics the in vivo physiology, the greater the chance for an IVIVC. Per its definition, IVIVC is a mathematical, functional relationship between selected in vitro results and selected biological parameters observed. For technical and analytical reasons, the in vitro setting may deviate from the physiology. The underlying principle is to ascertain high precision in the in vitro experiment in order to be able to detect changes in a product's performance. From a statistical point of view, the dissolution test has much more discriminatory power compared to the in vivo experiments given the innate variability in in vivo studies.

Compendial equipment is largely used for the in vitro part to facilitate the applicability of a Good Manufacturing Practices requirement. To establish point-to-point relationships, more than the classical single time point is required. Profiling of in vitro kinetics is mandatory. Depending on the subsequent algorithms of data reduction, a level of correlation can be established:

- Level A correlation is either based on PK modeling (e.g., Wagner-Nelson algorithm) or on a model-free approach (e.g., with the numerical deconvolution).

- Level B correlation uses the Statistical Moment Theory

- Level C correlation uses the classical PK parameters (i.e., $A \cup C, C_{\max }$, etc.)

In case multiple data points are used, the correlation may be reversible. At Level $A$, one batch may suffice to establish a correlation, whereas, for level $B$ and $C$, at least three batches need to be investigated.

Dr. Krämer emphasized that the attempts of establishing an IVIVC may fail due to several reasons. One reason for failing to establish an IVIVC can be the systemic absorption is the rate-limiting step, or that in vivo dissolution is not 
the rate-limiting step. Overlapping PK phenomena such as first pass effects or enterohepatic cycles may not allow proper analysis. Finally, the dissolution method is not discriminatory, which is considered a rare attribute after proper method development.

The next speaker, Dr. Erika Stippler, gave a presentation titled "USP Perspective on the Performance Verification Testing and Mechanical Calibration for USP Apparatus 1 and 2." Dr. Stippler emphasized the importance of the Performance Verification Testing (PVT) as required by the General Chapter $\langle 711\rangle$ to ensure a proper performance of Dissolution Apparatus 1 and 2. The assurance of the individual operational parameters of a dissolution apparatus is a prerequisite for a successful PVT.

Controlling the variability of the product is the goal of the regulators and the industry. The pharmacopeia contributes by standardizing the test methodology to reduce the variability arising from the procedure and the test equipment. As the purpose of the dissolution apparatus is to produce a standardized sample of the in vitro performance of the product, a logical expectation is for the apparatus to function in a standardized way. The USP Dissolution PVT serves to evaluate the holistic performance of dissolution test equipment by requiring the results for the reference standard tablets to fall within specified limits when tested under controlled standardized conditions. Hence, the PVT of dissolution apparatus by using a reference standard material is important to identify the potential sources of variability in dissolution results that are generated by the dissolution apparatus itself and the surrounding area.

Whereas the General Chapter $<711>$ has precisely defined values including tolerances for certain design and operational parameters of a dissolution apparatus, there are others such as the vessel dimensions, wobble measurement, or verticality measurements, that are not precisely defined. The contributions of the variability of the individual parameters as well as their combinations are evaluated with the PVT.

In the last 10 years, USP has published several studies in different journals that demonstrate the value of the PVT. Dr. Stippler presented the results of these studies in her presentation.

The next speaker was Dr. Xujin Lu, whose presentation was titled "Dissolution Testing for Evaluation of New Drugs in Controlled-Release Dosage Forms-Case Studies." In this presentation, he began with the ICH Q6A definitions for delayed-release and ER dosage forms, the two major types of controlled-release dosage forms, and described the requirements of the USP General Chapters $<711>$ and $<1092>$ for the dissolution testing of the two types of the dosage forms. For the delayed-release dosage forms, a two-part dissolution with an acid stage and a buffer stage was defined. For the ER dosage forms, at least three time points of drug release were specified. USP also provided specific acceptance tables for the interpretation of the dissolution results of the ER dosage forms (L1-L3) and the delayed-release dosage forms (A1A3 and B1-B3). Dr. Lu pointed to the US FDA dissolution method database and reviewed examples of the related dissolution methods. He further demonstrated different dissolution profiles and specification setting strategies from real cases, including from IR enteric-coated tablets, modified-release, enteric-coated tablets, overnight-ER formulations, and long-lasting (more than 50 days) ER injectable microspheres. Dr. Lu went through a case study in detail and discussed the challenges in the development of a two-part dissolution method for a formulation with enteric-coated beads in a capsule. He indicated that the two methods described in the USP (medium addition and medium exchange) both require unconventional operational procedures. Careful design and operation were necessary when performing either of the methods to maintain compendial compliance.

Professor Wei Wang (Tianjin Institute for Drug Control) gave a presentation titled "Critical Mechanical Calibration Parameters of Dissolution Apparatus." Professor Wang's presentation started with a description of the features of Mechanical Calibration (MC) versus PVT. Professor Wang also noted a study co-sponsored by FDA and PhRMR found that salicylic acid tablets were not adequately sensitive to dissolution Apparatus 1 and 2. Due to the weight variation of reference tablets and broad acceptance range of PVT, the minor mechanical difference between dissolution apparatus cannot be detected, although, the verification results using the calibrator meet the criteria. FDA published a guideline for industry on the use of $M C$ for dissolution Apparatus 1 and 2 in January 2010 along with a procedure (DPA-LOP-002). USP published dissolution toolkit procedures in March 2010 for MC and PVT for Apparatus 1 and 2. In 2013, the American Society for Testing Materials (ASTM) published a standard practice for the qualification of basket and paddle dissolution apparatus [E2503-13]. The International Conference on Harmonization (ICH) Q4B Annex 7(R2) endorsed the USP approach. The International Pharmaceutical Federation (FIP) also published a position paper on the qualification of paddle and basket dissolution apparatus. The China Pharmacopeia (CHP), 2015 edition, published General 
Requirements for Preparations <0931> Dissolution Test and Drug Release Test. The 2010 version of Dissolution Apparatus Self-Check/Verification Procedures is still being used, which indicates no mandatory requirements for MC in China.

Professor Wang reported a study that investigated the MC parameters using an RC8MD dissolution bath (Tianda Tianfa Technology Co., Tianjin China), USP prednisone tablet (R001B1), and testing procedures. The parameters evaluated include all those related to the FDA, USP, ASTM, and CHP specifications such as the temperature of dissolution medium, shaft rotational speed, depth, level, shaft verticality, vessel verticality, centering, shaft wobble, and basket wobble. The dissolution tests were performed using the parameters around the specified ranges. In order to complete all the tests, the instrument had to be disruptively adjusted to set some of the mechanical parameters. While the study had limitations, the results provided valuable information for evaluating the tolerance of the $\mathrm{MC}$ parameters and for identifying those high-risk parameters. Several remaining issues were pointed out by Professor Wang including vibration, paddle wobble, and issues with MC tools. It has been required by all domestic and foreign pharmacopeia that a dissolution test should be performed without any significant vibration. However, no specific data or appropriate criteria have been published. For paddle wobble, CHP 2015 General Requirement <0931> set a criterion of less than or equal to $0.5 \mathrm{~mm}$, which is very difficult to measure. It has been suggested that the paddle wobble can be reduced and controlled by setting a tight limit on the shaft verticality (within $90 \pm 0.2^{\circ}$ ). The issue of $\mathrm{MC}$ tools is critical. The most commonly used tools are single-task tools that are easy to obtain at low cost; some of them are possible to connect to a computer to record and store data. However, using the single-task tools takes a long time to complete the whole calibration; also; the manual operation may increase the risk of measurement errors and deviations. Integrated calibration tools with less manual operation and full connection with a computer are desirable, but are high in cost, require sophisticated design, certification, and user training. So far, no such universal and integrated tools are commercially available.

\section{Session 4}

After the lunch break, Session 4 started with Ms. Vivian Gray's presentation titled "FDA Requirements in Dissolution Testing for Evaluation and Approval of Generic Drugs in Solid Oral Dosage Forms, Including Similarity Factor." Her presentation included a sample of the Abbreviated New Drug Application particulars, especially the data requests and questions from FDA regarding the necessary bioequivalence and dissolution tests between the generic and Reference Listed Drug. She covered the new FDA guidance titled Dissolution Testing and Specification Criteria for Immediate-release Solid Oral Dosage Forms Containing Biopharmaceutics Classification System Class 1 and 3. This guidance gives especially helpful advice for developing methods for these two classes. This guidance does not help with BCS Classes II and IV as the dissolution is not rapid and is ratelimited. She examined the rules and problems with $f_{2}$. She discussed the problems in developing generic drug products briefly and suggested the Quality by Design (QbD) approach. Ms. Gray provided references to the QbD approach, use of IVIVC to optimize generic development, and a book titled "FDA Bioequivalence Standards."

The next speaker, Dr. Nikoletta Fotaki (University of Bath, UK), gave a presentation called "Biopharmaceutics for Generic Drug Products Development." She started her talk by reviewing the applications of dissolution testing. She discussed the physiological conditions and the physical parameters that can affect drug dissolution. Afterward, she talked about the biopharmaceutical considerations for dissolution media and hydrodynamics. She continued with the biopharmaceutical considerations for the selection of the dissolution medium based on the drug substance properties under fasted and fed state conditions and the dosage form properties (IR and MR formulations). She discussed the BCS-based biowaiver approach as described by the European Medicines Agency guideline and she highlighted the requirements from FDA and WHO guidelines. Then, she talked about the biopharmaceutical considerations for the dissolution testing of prolonged and delayed-release dosage forms from the recently issued European guideline for MR dosage forms, which requires establishing a link from in vitro release to PK parameters. She concluded her presentation with a summary of the key biopharmaceutical considerations during dissolution testing suggested from the regulators.

The next speaker was Dr. Xujin Lu, whose presentation was entitled "Biorelevant In-Vitro Dissolution for Solid Oral Dosage and Formulation Development." He began by enumerating the challenges in modern formulation development, especially for innovative drug development. The majority of new drug candidates are poorly soluble (BCS Class II or VI). Sophisticated approaches to formulation development are very often necessary to increase drug solubility and maximize drug bioavailability. The selection of appropriate formulation 
approaches and advanced drug delivery techniques is challenging. Adding to the challenges in early phase development are a limited quantity of drug substance and demanding timelines. Animal and human PK data are also limited in early development; therefore, the need has significantly increased for biorelevant in vitro evaluation of formulations. Formulation development relies on a good understanding of the API's physiochemical properties and biopharmaceutical properties, and also relies on a good understanding of the target profile of the drug product. The desired PK profile and the duration of action, projected human dose, and therapeutic range, food effect, and restrictions, and needs to combine with existing therapy, all need to be considered to establish a suitable formulation strategy. Biorelevant dissolution is one of the key experiments to guide the formulation development. It is a screening tool to test dissolutionbased hypotheses of prototype formulations; it can also predict bioavailability, and provide data for in silico modeling. Biorelevant dissolution plays a key role in the integrated risk assessment of biopharmaceutics and formulation development. Together, the in vitro, in silico, and in vivo studies ensures the understanding of the liabilities in drug development, guides the development of a suitable formulation strategy, enables decision making, and shortens development time.

Dr. Lu emphasized that the biorelevant in vitro dissolution used for early phase formulation development differs from the dissolution used for QC. The purpose of the former is for prediction of bioperformance not for ensuring batch-to-batch consistency. The devices for biorelevant dissolution can be compendial and non-compendial. The biorelevant media may not be the conventional buffers, but simulated fasted or fed state gastric and intestinal fluids. The method conditions of biorelevant dissolution can be universally chosen to mimic the GI tract in vivo. In contrast, the QC dissolution is more product specific, and the conditions are chosen to detect process and stability changes. The biorelevant dissolution does not require sink condition and full release of the drug, but only needs to show a rank order of the formulations. In later phase development, however, the biorelevant dissolution will be used to establish IVIVC, and ideally, the IVIVC and QC tests are expected to use the same dissolution method.

Continuing his presentation, Dr. Lu described some apparatus that have been used for biorelevant dissolution, providing examples in the compendial USP Apparatus 3 and Apparatus 4, and noncompendial transfer model, GI model, peristaltic model, crescent spindle model, biphasic model, and flux model, among others. He also summarized the biorelevant media and simulated biological fluids that have frequently been used for formulation development. He then presented two case studies, one for overcoming the $\mathrm{pH}$ effect in the development of a weakly basic drug. The other case was for food effect assessment in the development of a lipid tablet formulation. It was demonstrated, in both cases, that biorelevant in vitro dissolution testing is a useful and important tool for the formulation development and guide for the selection of appropriate formulation approaches.

Dr. Jian-Hwa Han gave the last talk, entitled "Practical Approach for Developing Dissolution Methods to Support Clinically Relevant Specifications." He started with the concept and approach for developing dissolution methods to support clinically relevant specifications. The activity has to be a product-specific with a well-designed development strategy at every drug development phase following QbD concepts. The intent is not trying to develop a generic, catch-all biorelevant dissolution method that can predict in vivo performance for all kinds of drug products.

Following the same method development concepts and strategies from the previous talk, Dr. Han introduced detailed method development practices at the different phases of drug development. Dr. Han stated it is not an expectation to explore too deep into the dissolution behavior for the drug product at the first-in-human study stage. At the proof-of-concept (POC) stage (i.e., Phase I/ Ila), we need to consider the drug product target profile and start the formulation design. It is never too early to explore the potential CMAs and CPPs. Moving to the next phase, the commercial formulation development (CFD) phase (i.e., Phase IIb/III), we need to exam the discriminating power of the dissolution method for the intended use. To improve the method as needed, this stage may involve the most work in method development. In this phase, it is good to identify the CMAs and CPPs and involve some exploratory DOE. It is important to examine the in vitro and in vivo drug release behavior for the target formulation and the "unordinary samples." If there is any concern about the dissolution method, it is strongly recommended to initiate an early communication with regulatory agencies regarding dissolution conditions and performance. At the last stage before filing (Phase III), the formulation/process and dissolution method are all finalized. The final stage is the time to wrap up all the learning and perform a confirmatory DOE against the CMAs and CPPs to ensure manufacturability and maintain BE. With all the available PK data, we will set up the appropriate dissolution specifications. Once again, 
the speaker emphasized the importance of collaboration within the multifunctional teams including analytical, formulation, and PK groups.

Dr. Han concluded that the ultimate goal for product development is always the benefit of the patient. The analytical team needs to know the dissolution method space, and choose the optimal condition for QC testing that can support clinically relevant specifications based on the clinical data. The formulation group must know the product space, and be able to establish a reliable control strategy to manufacture high-quality products consistently. The PK group guides the development with the true performance of the product (i.e., the clinical outcomes). The teamwork and collaboration will be the key to success for developing dissolution methods to support clinically relevant specifications.

\section{SUMMARY}

The workshop was very well attended with more than 400 attendees from industry, academia, and drug control and inspection agencies. In fact, the workshop registration was closed early because the attendance exceeded the capacity of the meeting facility. While the majority of the attendees were from China, a number of attendees were from the United States. Many attendees expressed interest in additional workshops on dissolution- and bioequivalence-related topics to provide additional educational and networking opportunities.
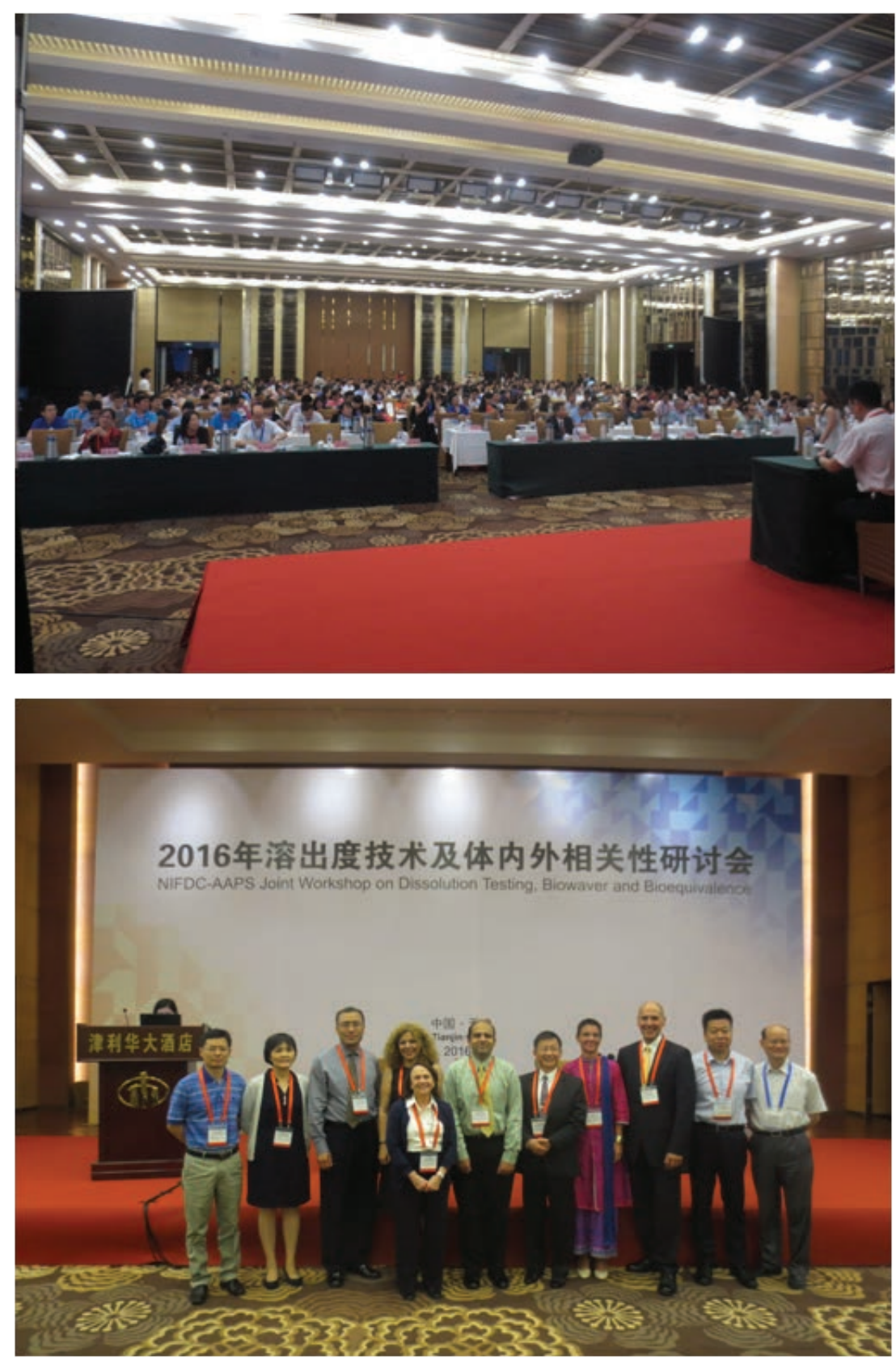

A view of the workshop meeting room during an ongoing session.

Workshop speakers and organizing committee members (left to right): Dr. Baoming Ning, Professor Yang Huaxin, Dr. Xujin Lu, Dr. Nikoletta Fotaki, Ms. Vivian Gray, Dr. Uppal Mushy, Dr. Jian-Hwa Han, Dr. Erika Stippler, Dr. Johannes Krämer, Professor Jianqiang Shao, and Professor Qiming Zhang. 